\title{
Direct Observation of Nonlocal Effects in a Superconductor
}

\author{
A. Suter, ${ }^{1, *}$ E. Morenzoni, ${ }^{1, \dagger}$ R. Khasanov, ${ }^{1,2}$ H. Luetkens, ${ }^{1,3}$ T. Prokscha, ${ }^{1}$ and N. Garifianov ${ }^{4}$ \\ ${ }^{1}$ Laboratory for Muon Spin Spectroscopy, PSI, CH-5232 Villigen PSI, Switzerland \\ ${ }^{2}$ Physics Institute, University of Zurich, CH-8057 Zurich, Switzerland \\ ${ }^{3}$ Institut für Metallphysik und Nukleare Festkörperphysik, TU Braunschweig, 38106 Braunschweig, Germany \\ ${ }^{4}$ Kazan Physical-Technical Institute, 420029 Kazan, Russian Federation
}

(Received 4 July 2003; published 26 February 2004)

\begin{abstract}
We have used the technique of low energy muon spin rotation to measure the local magnetic field profile $B(z)$ beneath the surface of a lead film maintained in the Meissner state ( $z$ depth from the surface, $z \lesssim 200 \mathrm{~nm}$ ). The data unambiguously show that $B(z)$ clearly deviates from an exponential law and represent the first direct, model independent proof for a nonlocal response in a superconductor.
\end{abstract}

Applying a small magnetic field parallel to the surface of a superconductor results in the expulsion of the magnetic flux from its interior, except for a small region on the nm scale close to its surface where the local field $B(z)$, measured at a depth $z$ from the surface, is heavily damped (Meissner-Ochsenfeld effect). The spatial field dependence $B(z)$ reflects the electromagnetic response of the superconductor and yields valuable information about its nature. If the Cooper pairs can be handled as pointlike, the electrodynamics of the system can be treated as local and the field extends exponentially over a typical length $\lambda$ (London penetration depth) of the order of a few tens to hundreds $\mathrm{nm}$. Nonlocal effects in superconductors arise when the variation of the electromagnetic field over the extent of the pairs cannot be neglected. This is, for instance, the case in conventional superconductors with coherence length $\xi \geqslant \lambda[1,2]$ or at nodes of the energy gap of unconventional superconductors, where the $k$-dependent coherence length becomes effectively infinite [3]. In $\mathrm{YBa}_{2} \mathrm{Cu}_{3} \mathrm{O}_{6.95}$ indications of nonlocal/ nonlinear effects have been found in the field dependence of the effective magnetic penetration depth as determined by muon spin rotation $(\mu \mathrm{SR})$ measurements in the bulk of the vortex lattice $[4,5]$.

In conventional superconductors the penetration profile of the magnetic field in the Meissner state is predicted to clearly deviate from the usual exponential decay at the surface. Though theoretical predictions are already half a century old, the direct experimental verification has been lacking. Signatures for nonlocal field penetration have been searched so far by induction techniques [6], magnetoabsorption resonance spectroscopy [7,8], and polarized neutron scattering reflectrometry (PNR) [9]. The pioneering measurements of Drangeid and Sommerhalder [6] revealed that there is a sign reversal of $B(z)$ (as predicted by theory), but no further quantitative results could be drawn from this experiment. The magnetoabsorption resonance spectroscopy technique uses the fact that qua- siparticles traveling parallel to the shielding current are bound to the surface by an effective magnetic potential. An indication of nonlocal effects in Al was inferred by comparing microwave induced resonant transitions between the energy levels of these bound states with transition fields calculated from the energy levels of the trapping potential, parametrized to include the shape of the nonlocal BCS-like potential [7,8]. Because of the resonant character of the experiment, only a few specific points of the potential are probed. In addition the normal metallic state has to be understood very well in order to interpret the data. This, together with uncertainties in modeling the surface bound states, makes the confirmation of the predicted functional form of $B(z)$ rather indirect. The specular reflectivity of neutrons spin polarized parallel or antiparallel to $\boldsymbol{B}$ also depends on the field profile. However, this technique requires model fitting of spin-dependent scattering intensities rather than giving a direct measure of the spatial variation of the magnetic field. Up to now nonlocal corrections have been found to lie beyond the sensitivity of PNR [9].

A direct measurement of $B(z)$ requires experimental probes that allow one to measure microscopically the magnetic properties of the region extending only a few tens of nm away from the surface. We used the newly developed low energy muon spin rotation technique (LE- $\mu \mathrm{SR}$ ) to map $B(z)$ in superconducting lead [10]. We find that the functional dependence of $B(z)$ is indeed nonexponential and that it follows the predicted PippardBCS theory $[1,2,11-13]$. We obtain a value for the London penetration depth $\lambda_{\mathrm{L}}=57(2) \mathrm{nm}$ and a clean-limit coherence length $\xi_{0}=90(5) \mathrm{nm}$ for $\mathrm{Pb}$.

A weak external magnetic field acts on the ground state of the superconductor as a perturbation. Within a perturbation expansion one can show [14-16] that the following nonlocal relation between the supercurrent density $\boldsymbol{j}$ and the vector potential $\boldsymbol{A}$ holds (in Coulomb gauge $\nabla \cdot \boldsymbol{A}=0)$ : 


$$
j_{\alpha}(\boldsymbol{r})=-\sum_{\beta} \int \underbrace{\left[R_{\alpha \beta}\left(\boldsymbol{r}-\boldsymbol{r}^{\prime}\right)-\frac{e^{2} n_{\mathrm{S}}}{m^{*}} \delta\left(\boldsymbol{r}-\boldsymbol{r}^{\prime}\right) \delta_{\alpha \beta}\right]}_{=: K_{\alpha \beta}\left(\boldsymbol{r}-\boldsymbol{r}^{\prime}\right)} A_{\beta}\left(\boldsymbol{r}^{\prime}\right) d \boldsymbol{r}^{\prime},
$$

where $e$ is the electron charge, $n_{\mathrm{S}}$ the supercarrier density, $m^{*}$ the effective electron mass, and $\nabla \wedge \boldsymbol{A}=\boldsymbol{B}$. The first term in the square brackets, $R_{\alpha \beta}$, describes the paramagnetic response, whereas the second reflects the diamagnetic one. $K_{\alpha \beta}$ is called the kernel. If the wave function of the electronic ground state were "rigid" with respect to all perturbations (rather than only those which lead to transverse excitations) $R_{\alpha \beta}$ would be identically zero and Eq. (1) would reduce to the local $\boldsymbol{j}$ - $\boldsymbol{A}$ relation

$$
j_{\alpha}(\boldsymbol{r})=-\frac{1}{\mu_{0} \lambda_{\mathrm{L}}^{2}} A_{\alpha}(\boldsymbol{r})
$$

with $\mu_{0}$ the magnetic permeability of the vacuum. This combined with the Maxwell equation $\nabla \wedge \boldsymbol{B}=\mu_{0} \boldsymbol{j}$ yields, at a plane superconductor-vacuum interface, the result of an exponentially suppressed magnetic field

$$
B(z)=B_{\text {ext }} \exp \left(-z / \lambda_{\mathrm{L}}\right)
$$

with the London penetration depth $\lambda_{\mathrm{L}}=\sqrt{\frac{m^{*}}{\mu_{0} e^{2} n_{\mathrm{S}}}}$, which is the well-known result.

However, $R_{\alpha \beta}$ has a range of the order of the diameter of the Cooper pairs, i.e., of the coherence length $\xi$. The magnetic penetration depth sets the length scale for the decay of the magnetization; for $\lambda \gg \xi$ the spatial variation of the vector potential $\boldsymbol{A}$ over the superconducting pairs is negligible and the one-parameter local description of Eq. (2) holds. If $\xi \gtrsim \lambda$ the full nonlocal description has to be taken into account. Fourier analysis of the perturbation and of the response show that [17]

$$
B(z)=B_{\text {ext }} \int \frac{q}{q^{2}+\mu_{0} K(q \xi, T, \ell)} \sin (q z) d q,
$$

where $q$ is the wave vector, $T$ is the temperature, and $\ell$ is the electron mean free path. The functional form of $K(q \xi, T, \ell)$ [Fourier transform of the kernel in Eq. (1)], starting from microscopic considerations, is explicitly known in the weak (BCS) $[2,13]$ and the strong coupling limit $[11,12,18]$. The resulting formulas are rather involved but are very close to the phenomenological expression of Pippard [1,15],

$$
\mu_{0} K(x, T, \ell)=\frac{1}{\lambda_{\mathrm{L}}^{2}} \frac{\xi(T, \ell)}{\xi(0, \ell)}\left[\frac{3}{2} \frac{1}{x^{3}}\left\{\left(1+x^{2}\right) \arctan (x)-x\right\}\right]
$$

with $x=q \xi(T, \ell)$ and $\frac{1}{\xi(T, \ell)}=\frac{J(0, T)}{\xi_{0}}+\frac{1}{\ell}$, where $\xi_{0}$ is the clean-limit coherence length and $J(0, T)$ is given according to Ref. [2]. The kernel has the property $\mu_{0} K(x \rightarrow$ $0, T \rightarrow 0)=\lambda_{\mathrm{L}}^{-2}$ (corresponding to the local limit). This holds for the BCS kernel as well.
Figure 1 shows the theoretical predictions in the case that $\xi \gg \lambda$. The main features can be understood qualitatively. In the nonlocal case the perturbing field changes over the extension of the Cooper pairs; since the charges within a Cooper pair do not experience the same force, the screening response is less effective and hence the field falls initially less rapidly than in the case of a point response. With the field penetrating further, at some range Cooper pairs "overcompensate," which accounts for the curvature of $\log [B(z)]$ as well as for the field reversal of $B(z)$ [6].

We have used the $100 \%$ polarized low energy muon beam at the Paul Scherrer Institute and the muon spin rotation technique to directly determine the values of the magnetic field as a function of depth underneath the surface [10]. With a tunable energy between 0.5 and $30 \mathrm{keV}$ these particles are implanted one at a time at variable depth between $\sim 1 \mathrm{~nm}$ and a few hundreds $\mathrm{nm}$ beneath the surface of the specimen. The local magnetic field $B(z)$ at the stop position causes the muon spin to precess. The temporal evolution of the spin polarization of the muon ensemble, $P(t)$, is monitored by the detection of the decay positrons which are anisotropically emitted preferentially in the direction of the muon spin at the moment of the decay [19]. This quantity is directly related by a Fourier transform to the internal magnetic field

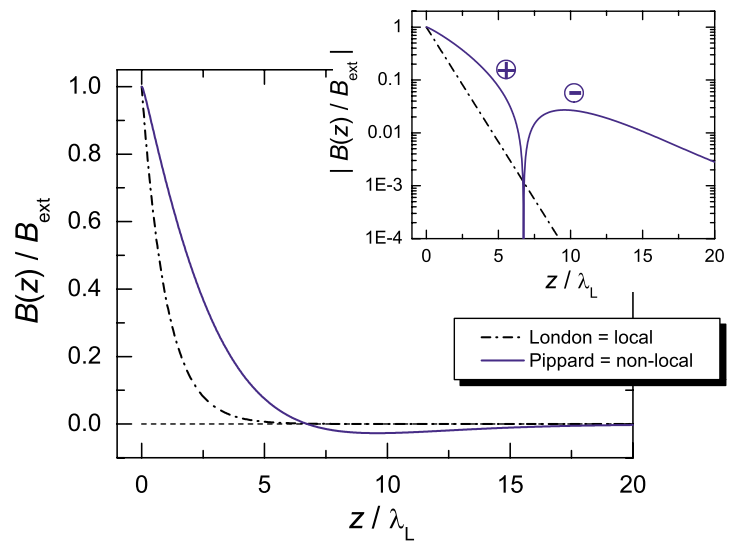

FIG. 1 (color online). Comparison between local and nonlocal magnetic penetration profiles for the same $\lambda_{\mathrm{L}}$ (parameters from Al for $T \rightarrow 0, \lambda_{\mathrm{L}}=18 \mathrm{~nm}$, and $\xi_{0}=1600 \mathrm{~nm}$ ). The dash-dotted line shows the typical exponential field profile predicted from the London theory. The solid line shows the nonlocal field profile from BCS theory. Specific features are (i) the penetration profile is nonexponential, (ii) the initial slope is less steep than in the local approximation, (iii) there is a field reversal before $B(z)$ decays towards zero, and (iv) the inset shows that $\log [B(z)]$ vs $z$ exhibits a clear curvature. 
distribution sensed by the muon ensemble. The field distribution $p(B)$ is connected to the implantation profile of the muons $n(z, E)$ by

$$
n(z, E) d z=p(B) d B,
$$

which states that the probability that a muon will experience a field in the interval $[B, B+d B]$ is given by the probability that it will stop at a depth in the range $[z, z+$ $d z$ ]. Integrating Eq. (6) on both sides yields

$$
\int_{0}^{z} n(\zeta, E) d \zeta=\int_{B}^{\infty} p(\beta) d \beta,
$$

which, for a chosen $z$, is an equation for $B$. We calculate $n(z, E)$ with the Monte Carlo code TRIM.SP [20], which yields reliable implantation profiles for the muons, as shown in Ref. [21]. Since $n(z, E)$ is known and $p(B)$ is measured, $B(z)$ can be uniquely determined.

To search for nonlocal effects we investigated thin films of type-I superconducting $\mathrm{Pb}$. The films were sputtered directly onto sapphire or quartz crystals mounted on a He flow cryostat. The samples had a diameter of $50 \mathrm{~mm}$ and a thickness of 1055(50) nm (sample I) and 430(20) nm (sample II) as determined by a high sensitivity surface profiler and Rutherford backscattering (RBS). For sample I an oxide layer of 5.8(3) $\mathrm{nm}$ was found by RBS, whereas sample II had an oxide layer of 16(2) $\mathrm{nm}$. The critical temperature was determined by means of resistivity and susceptibility measurements to $T_{\mathrm{c}}=7.21(1) \mathrm{K}$. The mean free path was estimated to be $\simeq 100 \mathrm{~nm}$ from resistivity measurements. After zero field cooling a $B_{\text {ext }}=8.82(6) \mathrm{mT}$ was applied parallel to the surface. In the experiment low energy muons, $\mu^{+}$, with their spin perpendicular to the magnetic field and their momentum were implanted in the samples at a variable depth up to $150 \mathrm{~nm}$ and the decay positrons detected by scintillation counters surrounding the sample. Details and characteristics of the low energy $\mu^{+}$source and spectrometer are given in [22]. The distribution $p(B)$ was derived by maximum entropy Fourier analysis of the muon spin precession frequency of the decay positron histograms [23-25].

The measured magnetic field penetration profiles $B(z)$ in the Meissner state of $\mathrm{Pb}$ at $T=3.05(3) \mathrm{K}$ and $T=$ 6.66(3) K, respectively, are shown in the Figs. 2 and 3 (sample I). Sample II gives consistent results. The curves show clear deviations from the exponential behavior with the characteristic curvature. Qualitatively, the initial slope of the curve is determined by $\lambda$, whereas the $\log [B(z)]$ curvature is mainly governed by the ratio $\xi / \lambda$. The deviations are more pronounced at lower temperature: on approaching $T_{\mathrm{c}}$ the superconductor becomes more and more local. This is a consequence of the fact that $\lambda$ has a pronounced temperature dependence close to $T_{\mathrm{c}}$, but $\xi$ has not. The reversal of the penetrating field could not be detected since the muon range in the experiment was not

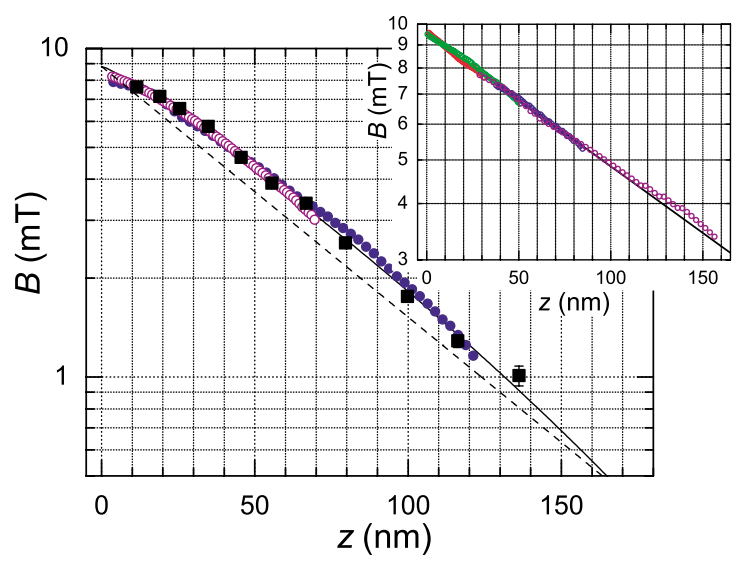

FIG. 2 (color). Measured magnetic penetration profile $B(z)$ of $\mathrm{Pb}$ in the Meissner state for $T=3.05 \mathrm{~K}\left[T_{\mathrm{c}}=7.21(2) \mathrm{K}\right]$ in an external field of 8.82(6) $\mathrm{mT}$, applied parallel to the surface of the film. Implantation energies $E$ of the muons: $5.2 \mathrm{keV}$ (open circles) and $14.8 \mathrm{keV}$ (closed circles). The mean values are plotted as closed squares with implantation energies $(2.5,4.0$, $5.4,7.5,10.0,12.4,15.0,18.0,22.5,26.0,30.0) \mathrm{keV}$ from left to right. The solid curve is a fit according to Eq. (4) with the BCS parameters $\lambda_{\mathrm{L}}=57(2) \mathrm{nm}, \xi_{0}=90(5) \mathrm{nm}$, and $\ell=100 \mathrm{~nm}$ (fixed). The dashed curve shows the exponential dependence assuming the values from the full BCS fit. The inset displays results of Meissner state measurements of optimally doped $\mathrm{YBa}_{2} \mathrm{Cu}_{3} \mathrm{O}_{7-\delta}$ at $T=20 \mathrm{~K}\left(T_{\mathrm{c}}=87.5 \mathrm{~K}\right)$. This curve is purely exponential, as expected.

large enough. We point out that from Eq. (7) it follows that the functional relationship $B(z)$ can be determined from overlapping implantation profiles $n(z, E)$ obtained at different energies. In order not to overload the figures only

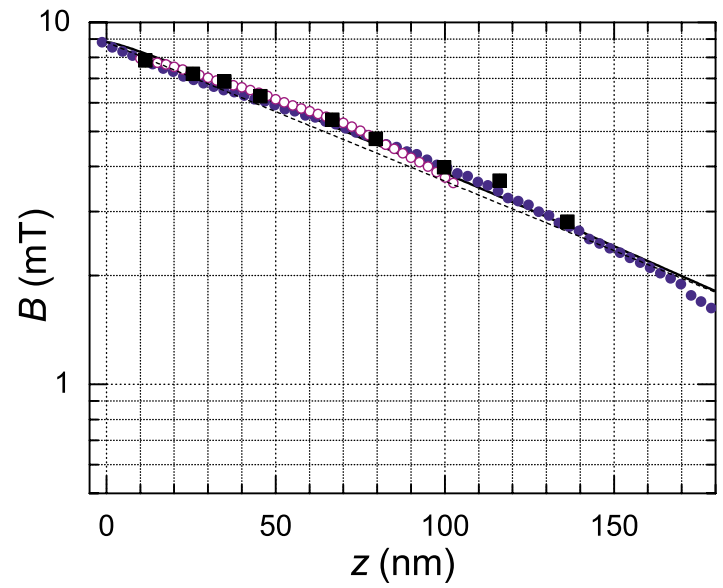

FIG. 3 (color). Magnetic penetration profile $B(z)$ of $\mathrm{Pb}$ in the Meissner state for $T=6.66 \mathrm{~K}\left[T_{\mathrm{c}}=7.21(2) \mathrm{K}\right]$ in an external field of 8.82(6) $\mathrm{mT}$, applied parallel to the surface of the film. Implantation energies of the muons: $14.8 \mathrm{keV}$ (open circles) and $30.0 \mathrm{keV}$ (closed circles). The mean values are shown as closed squares with implantation energies $(2.5,5.4,7.5,10.0,15.0$, 18.0, 22.5, 26.0, 30.0) $\mathrm{keV}$. The solid curve is a fit according to BCS theory, whereas the dashed curve shows an exponential with the same $\lambda_{\mathrm{L}}$. 
some energies are shown (displayed in different colors). Figure 2 shows, for instance, curves obtained with 5.2 and $14.8 \mathrm{keV}$ data. Their small relative deviation is a measure of the uncertainty in the derivation of $B(z)$ and is an important self-consistency test of the reliability of the results. For a more detailed discussion and a compilation of additional data we refer to a forthcoming publication. Figures 2 and 3 also present the results from an alternative analysis using the mean values for $\langle B\rangle=$ $\int B p(B) d B$ and $\langle z\rangle=\int z n(z, E) d z$. The points $\langle B\rangle$ plotted versus $\langle z\rangle$ are in very good agreement with the curve $B(z)$ obtained from Eq. (7). As a further cross-check for the sensitivity and accuracy of the method based on Eq. (7) we reanalyzed profiles in the Meissner state of optimally doped $\mathrm{YBa}_{2} \mathrm{Cu}_{3} \mathrm{O}_{7-\delta}$ at $T=20 \mathrm{~K}\left(T_{\mathrm{c}}=87.5 \mathrm{~K}\right.$, [26]). In this extreme type-II superconductor, at this temperature, the data perfectly follow an exponential law as expected for the local electrodynamic response (Fig. 2 inset) and are also in very good agreement with the results previously obtained by an iterative solution of Eq. (6). Analyzing the $\mathrm{Pb}$ data with the BCS and Pippard theory leads to consistent results for $\lambda_{\mathrm{L}}$ and $\xi_{0}$. The strong electron-phonon coupling in $\mathrm{Pb}$ can be accounted for by a renormalization of the weak coupling parameters of the form $\lambda_{\mathrm{BCS}} \rightarrow \lambda_{\mathrm{L}} / \sqrt{Z}$ and $\xi_{\mathrm{BCS}} \rightarrow \xi_{0} Z$ with $Z=$ $1+\lambda_{\mathrm{e}-\mathrm{ph}} \approx 2.55$ for $\mathrm{Pb}$ [27] ( $\lambda_{\mathrm{e} \text {-ph }}$, the electron-phonon coupling). Compiling all the data, we find a magnetic penetration depth $\lambda_{\mathrm{L}}=57(2) \mathrm{nm}$ and a coherence length $\xi_{0}=90(5) \mathrm{nm}$ for $T=0 \mathrm{~K}$.

In conclusion, by using spin polarized muons of a few $\mathrm{keV}$ energy as surface sensitive magnetic microprobes, we have shown that the magnetic penetration profile at the surface of superconducting $\mathrm{Pb}$ is nonexponential and that nonlocal electrodynamics effects, as predicted by Pippard and BCS theory, are responsible for this behavior. We believe that the ability to measure magnetic profiles beneath surfaces and buried interfaces on the $\mathrm{nm}$ scale, by means of LE- $\mu \mathrm{SR}$, opens the door to explore interesting systems from a fundamental as well as from an applied point of view. We look forward to further measurements of magnetic fields and fluctuations in surface superconductivity (e.g., proximity effects in multilayers, surface sheath) and magnetism.

This work was performed at the Swiss Muon Source $(\mathrm{S} \mu \mathrm{S})$, Paul Scherrer Institute, Villigen, Switzerland. We thank M. Horisberger for the preparation of the samples, M. Doebeli for the RBS measurements, and D. Eshchenko and D. Ucko for the help during part of the measurements.

*Electronic address: andreas.suter@psi.ch
${ }^{\dagger}$ Electronic address: elvezio.morenzoni@psi.ch

[1] A. B. Pippard, Proc. R. Soc. London, Ser. A 216, 547 (1953).

[2] J. Bardeen, L. N. Cooper, and J. R. Schrieffer, Phys. Rev. 108, 1175 (1957).

[3] I. Kosztin and A. J. Leggett, Phys. Rev. Lett. 79, 135 (1997).

[4] J. E. Sonier, J. H. Brewer, R. F. Kiefl, G. D. Morris, R. Miller, D. A. Bonn, J. Chakhalian, R. H. Heffner, W. N. Hardy, and R. Liang, Phys. Rev. Lett. 83, 4156 (1999).

[5] M. H. S. Amin, M. Franz, and I. Affleck, Phys. Rev. Lett. 84, 5864 (2000).

[6] K. E. Drangeid and R. Sommerhalder, Phys. Rev. Lett. 8, 467 (1962).

[7] R. E. Doezema, J. N. Huffaker, S. Whitmore, J. Slinkman, and W. E. Lawrence, Phys. Rev. Lett. 53, 714 (1984).

[8] R. E. Doezema, S. C. Whitmore, and J. N. Huffaker, Phys. Rev. B 34, 4614 (1986).

[9] M. P. Nutley, A. T. Boothroyd, C. R. Staddon, D. M. Paul, and J. Penfold, Phys. Rev. B 49, 15789 (1994).

[10] E. Morenzoni, F. Kottmann, D. Maden, B. Matthias, M. Meyberg, T. Prokscha, T. Wutzke, and U. Zimmermann, Phys. Rev. Lett. 72, 2793 (1994).

[11] S. B. Nam, Phys. Rev. 156, 470 (1967).

[12] S. B. Nam, Phys. Rev. 156, 487 (1967).

[13] J. Halbritter, Z. Phys. 243, 201 (1971).

[14] J. R. Schrieffer, Theory of Superconductivity (AddisonWesley, San Francisco, 1964).

[15] M. Tinkham, Introduction to Superconductivity (Krieger, New York, 1980).

[16] C. P. Poole, H. A. Farach, and R. J. Creswick, Superconductivity (Academic Press, San Diego, 1995).

[17] This expression is valid for specular reflection of the charge carriers at the surface. The case of diffuse scattering has been studied analytically and numerically and shows only minor differences for relevant parameters such as the penetration depth.

[18] J. C. Swihart and W. Shaw, Physica (Utrecht) 55, 678 (1971).

[19] S. J. Blundell, Contemp. Phys. 40, 175 (1999).

[20] W. Eckstein, Computer Simulation of Ion-Solid Interactions (Springer, Berlin, 1991).

[21] E. Morenzoni, H. Glückler, T. Prokscha, R. Khasanov, H. Luetkens, M. Birke, E. M. Forgan, C. Niedermeyer, and M. Pleines, Nucl. Instrum. Methods Phys. Res., Sect. B 192, 254 (2002).

[22] E. Morenzoni et al., Physica (Amsterdam) 289B-290B, 653 (2000).

[23] J. Skilling and R. K. Bryan, Mon. Not. R. Astron. Soc. 211, 111 (1984).

[24] B. D. Rainford and G. J. Daniell, Hyperfine Interact. 87, 1129 (1994)

[25] T. Riseman and E. M. Forgan, Physica (Amsterdam) 289B-290B, 718 (2000).

[26] T. J. Jackson et al., Phys. Rev. Lett. 84, 4958 (2000).

[27] J. P. Carbotte, Rev. Mod. Phys. 62, 1027 (1990). 